Im Journal Club dieser Ausgabe der pädiatrie hautnah referieren und kommentieren diese Experten für Sie Arbeiten aus der internationalen Fachliteratur.
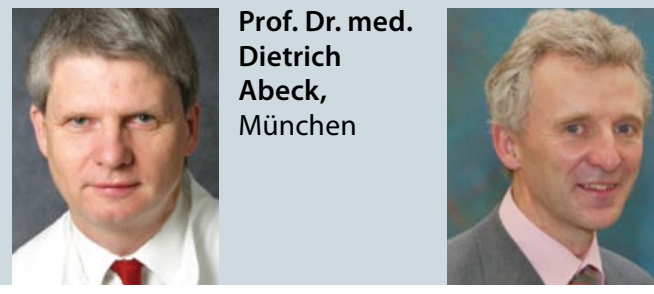

Dr. med. Martin Claßen, Bremen

\section{CPAP-Pausen sind nachteilig beim Entwöhnen}

Wie sollte man beim Absetzen eines CPAP vorgehen, damit der Einsatz von CPAP und Sauerstoff sowie das Risiko einer Bronchopulmonalen Dysplasie (BPD) oder der Krankenhausaufenthalt minimiert wird? Eine Studie aus Australien gibt nun Klarheit.

B ei den Bemühungen, das Barotrauma der Lunge zu minimieren, die Risiken der mechanischen Ventilation sowie Apnoen bei unreifen Frühgeborenen zu vermeiden, spielt der nasal applizierte kontinuierliche positive Atemwegsdruck (CPAP) die Hauptrolle auf neonatologischen Intensivstationen. Unklarheiten gibt es nicht mehr so sehr mit der Indikationsstellung als über die beste $\mathrm{Me}$ thode, den Kindern die CPAP-Unterstützung abzutrainieren.

Hierzu wurden in Australien in drei großen Kliniken 177 Kinder < 30 SSW in drei Gruppen randomisiert: In Gruppe 1 wurde nach Erreichen bestimmter Stabilitätskriterien (Tabelle) die CPAPAtemhilfe dauerhaft beendet. In Gruppe ren konnte.
2 wurden zunächst Pausen eingeführt, die jeweils wieder von sechsstündigen CPAP-Phasen abgelöst wurden. Die Pausen wurden um 2-4-Stunden-Phasen schrittweise verlängert. Die Kinder der Gruppe 3 wurden wie in Gruppe 2 schrittweise abtrainiert, erhielten aber in den CPAP-Pausen noch einen zusätzlichen nasalen Gasstrom mit 0,5 1/min über eine Art Nasenbrille, die aber keinen erhöhten Atemwegsdruck generie-

Die beiden allmählichen Methoden in Gruppe 2 und 3 waren dabei signifikant unterlegen, insbesondere was die Gesamtdauer der Atemhilfe (Gruppe 1: 24,4 Tage; 2: 38,6Tage und 3: 30,5 Tage; $\mathrm{p}<0,0001$ ), aber auch die Dauer des zu-

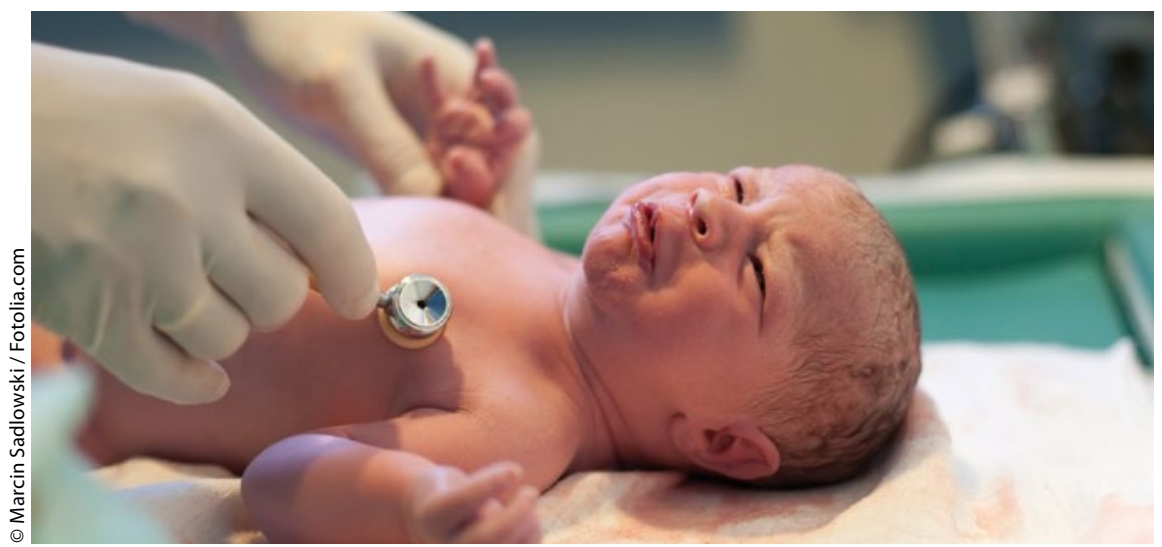

Die besten Werte zeigt das Neugeborene, wenn die CPAP-Atemhilfe nicht stufenweise, sondern abrupt beendet wird.

\section{Stabilitätskriterien für die Tabelle Beendigung der CPAP-Atemhilfe}

Alle acht Kriterien müssen für die letzten 12 Stunden erfüllt sein:

- CPAP 4-6 $\mathrm{cm} \mathrm{H}_{2} \mathrm{O}$

- O2-Bedarf $<25 \%$ und nicht ansteigend

- Atemfrequenz <60/min

_ keine signifikanten Einziehungen

_ weniger als drei Episoden mit selbstlimitierenden Apnöen (<20 sec) und/oder Bradykardien (<100/min) und/oder Sättigungsabfälle $(\leq 86 \%)$ innerhalb einer Stunde während der letzten 6 Stunden

_ mittlere Sättigung $>86 \%$ in der meisten Zeit oder transkutaner $\mathrm{PaO}_{2}>45 \mathrm{mmHg}$

_ keine laufende Behandlung für PDA oder Sepsis

_ Tolerieren kurzer CPAP-Pausen während der pflegerischen Versorgung (bis zu 15 min)

sätzlichen Sauerstoffbedarfs und die Dauer des Klinikaufenthaltes angeht. Auch zeigt der Trend der BPD-Entwicklung einen Vorteil für die Gruppe 1.

Todd DA et al. Methods of weaning preterm babies $<30$ weeks gestation off CPAP: a multicentre randomised controlled trial. Arch Dis Child Fetal Neonatal Ed 2012; 97: F236-40

Kommentar: Wissenschaftlich erfreulich ist, wie viele Verfahren mittlerweile in der Neonatologie evidenzbasiert anzuwenden sind. Dass diesmal die sanften, allmählichen Methoden nachteilig waren, hatte ich so nicht erwartet. Umso besser, dass nun mehr Klarheit herrscht. Für neonatologische Einheiten sei die allmähliche Reduktion des CPAP-Druckniveaus, der Gebrauch der Zielkriterien zur Beendigung des CPAP und die Kriterien zum Wiederbeginn, die in der Publikation detailliert aufgeführt sind, zur alltäglichen Anwendung als Handlungsanweisung empfohlen.

Dr. Martin Claßen 